\title{
Does daily travel pattern disclose people's preference?
}

\author{
Mingxin Gan* \\ Donlinks School of Economics \\ and Management, \\ University of Science and \\ Technology Beijing, China \\ ganmx@ustb.edu.cn
}

\author{
Ling Gao \\ Donlinks School of Economics \\ and Management, \\ University of Science and \\ Technology Beijing, China \\ gao.ling321@163.com
}

\author{
Yang Han \\ Donlinks School of Economics \\ and Management, \\ University of Science and \\ Technology Beijing, China \\ dozz-yico@163.com
}

\begin{abstract}
Existing studies normally focus on extracting temporal or periodical patterns of people's daily travel for location based services. However, people's characteristics and preference are actually paid much more attention by business. Therefore, how to capture characteristics from their daily travel patterns, is an interesting question. In order to address the research question, we first develop two basic measures in terms of repetitiveness of travel and then two advanced measures, to capture people's activity of daily travel, and the colorfulness of lifestyle, respectively. Incorporating historical trajectories, with real-time positions from a location-based social network (LBSN), i.e. Foursquare, we conduct statistical analysis for people's travel patterns in US cities. Finally, we illustrate people's profiles of travel patterns and lifestyles. Results show that people's preference can be inferred from the developed activity and colorfulness measures. Those findings demonstrate that proposed measures are supposed to be effectively adopted for researchers on travel pattern analysis and preference analysis, and further give suggestions to individuals for location-based decision making.
\end{abstract}

\section{Introduction}

The popularity of smart devices and mobile applications leads to a rapid emergence of locationbased social networks (LBSN) which enable us to get geographic information of location resources.

Existing studies normally focus on extracting temporal or periodical patterns of people's daily travel.
People's potential demand and relatively stable taste are usually illustrated by exploiting measurable relations between people and locations from historical visits, and people's preference similarities are therefore captured from travel trajectories and characteristics [16]. Studies on travel habits and patterns are mainly used to predict trajectory [7-14] which is normally conducted via modeling GPS's trajectories [15-16] or analyzing LBSN-based check-in data [11-14].

Although people's travel characteristics can be exploited from trajectories, existing studies have not been aware that people's final destinations mainly depend on their distinctive personality characteristics or various daily lifestyles which are influenced by not only psychological, gender, professional characteristics but also environmental factors of different cities. On the other hand, in practical, most city managers pay more attention to people's daily lifestyle. Besides, people's personality characteristics and preference are concerned by various business fields. However, no attempt is made to analyze people's characteristics or lifestyle from their related daily travel patterns. Therefore, how to evaluate people's personality characteristics and their lifestyle, from their observed daily travel, are both interesting questions.

To overcome these limitations, in this study, we answer the following research questions:

(1) How to evaluate activity of people's daily travel patterns?

(2) How to evaluate colorfulness of people's daily lifestyle?

(3) Does activity or colorfulness disclose people's preference? 

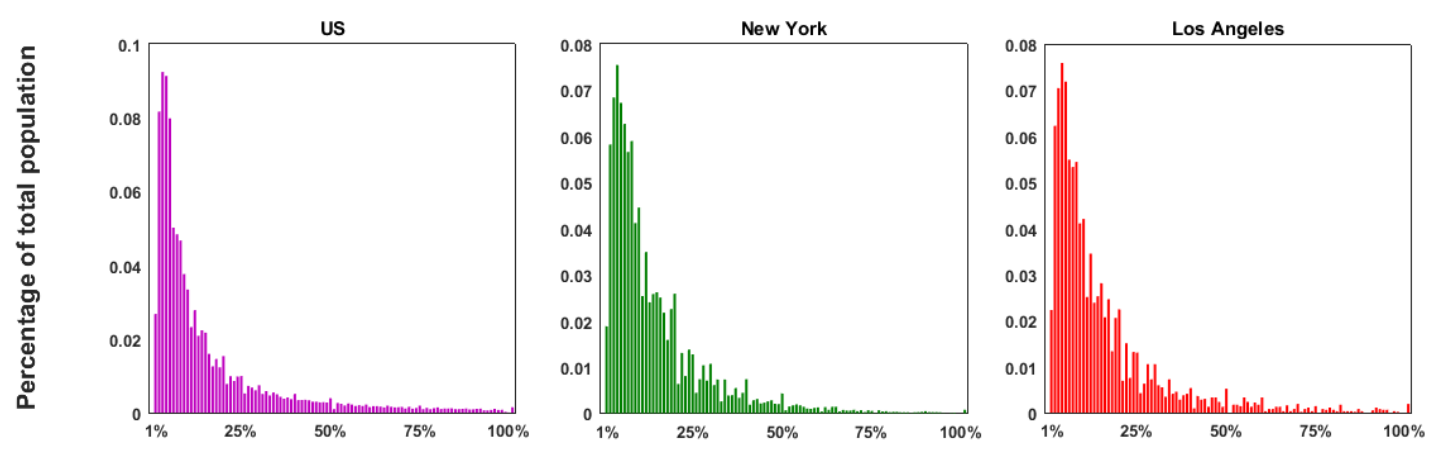

Recurrence ratio

Figure 1. Distribution of the recurrence ratio in location in terms of percentages of total population in US cities.

To answer these research questions, we sample a dataset crawled from Foursquare, which contains more than 30 million check-ins from more than 400 cities in 84 countries. We concentrate on US cities. The original data included 3,545,288 check-ins of 50,812 people for 501,415 POIs (Point Of Interest). Each check-in has time-stamp and such geographic information as longitude, latitude and city ID. We apply statistical analysis to the data samples and begin by grouping all geographic locations with category tags. Then for each individual whose visiting records are extracted from the data, we obtain all geographic locations visited by him/her described by corresponding longitude and latitude with category tags added. Besides, we also incorporate the time-stamp of each visiting record.

Contributions of the paper are summarized as below:

(i) Rather than extracting the temporal or periodical patterns of people's daily travel trajectories in existing studies, we propose a set of novel measures for capturing and evaluating people's daily travel patterns as well as their personality characteristics in lifestyle, in terms of repetitiveness on locations and categories. Investigation based on the statistical analysis and comparison of the developed measures are conducted with respect to people in US cities.

(ii) We illustrate people's profiles using their total historical daily travel records tagged with location categories, to investigate whether people's daily travel patterns and their personality characteristics in daily lifestyle, in terms of the developed measures activity and colorfulness, can be used to distinguish their preference on daily travel. Findings demonstrate that both people's daily travel pattern and personality characteristics of lifestyle disclose their location preference.

\section{Study 1: Is people active in daily travel?}

We develop a set of measures to evaluate whether a person is active or not in terms of their daily travel patterns. We first propose a basic measure named the recurrence ratio at locations to find people's repetitiveness of their daily travel. Then we give an advanced novel measure activity to justify the degree of repetitiveness which reflects people's personality characteristics of travel patterns.

\subsection{Recurrence ratio at locations}

We first define the recurrence ratio at location as the ratio of an individual's visits on a location among all his/her visits in a same area (a city or a country). First, we calculate numbers of locations where an individual visit at a frequency within the repetition internal. Then, people who visited at least one location within the recurrence ratio interval are added. Then we summarize the total number of people grouped by intervals of the recurrence ratio at location in Figure1. Finally, we obtain the distribution of the recurrence ratio at location in intervals by number of people in a certain area. By averaging number of locations people visited in a certain interval of the recurrence ratio in an area, we develop another variable named average number of locations in recurrence ratio, for a city and for US. We observe that the variables average number of locations in recurrence ratio, exhibit Gauss distributions with a consistency for US cities and US as a whole area.

The average number of locations at each internal of the recurrence ratio is summarized in Figure 3. On average, people visit about 1-2 locations within a very low recurrence ratio of $0-1 \%, 2-3$ locations within the internal of $2-4 \%$, and $1-2$ locations within $4-5 \%$, 


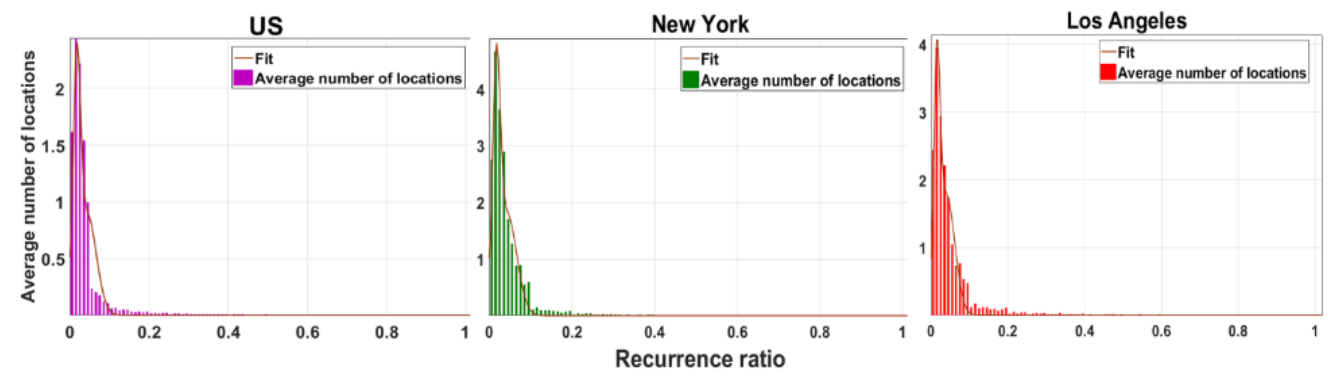

Figure 2. Distribution of the average recurrence ratio at location in US cities.

respectively, in US. The average number of locations monotonically decreases along with the increase of the recurrence ratio. We find the recurrence ratio at location follow an exponential distribution in terms of the average number of locations in each US city.

We then define a related measure named average recurrence ratio at locations for an individual in the area to reflect people's visiting repetitiveness across his/her daily travel, to which the number of his/her appearances in the internals of the recurrence ratios equal or below, is as the same as the number of his/her appearances in the internals of the recurrence ratios beyond. By averaging the average recurrence ratio at location for all people in the area, we get the average recurrence rate at location for the area.

Ave_recur_loc $i j=a$ ratio for individual $j$ in city $i$, to which \# $j$ 's appearances in the internals of recurrence ratios below or equal, equal to \# $j$ 's appearances in the internals of recurrence ratios beyond.

Ave_recur $i=$ a ratio for city $i$, to which \# people in the internals of recurrence ratios at locations below or equal is as the same as \# people in the internals of recurrence ratios at locations beyond, being calculated by averaging Ave_recur_loc $i j$ for all people in city $i$.

We illustrate the distribution of per average recurrence ratio at location in terms of the number of people in US in Figure 2. Result shows that few people have very large recurrence ratios on locations, for example, a workaholic goes to his working place every day with a high recurrence ratio of $90 \%$.

We also investigate the average recurrence ratio at location in terms of individuals, locations and categories, respectively, and illustrate the result in Table 1 and Figure 3. Taking the whole dataset as samples, on average, an individual's visits have a recurrence ratio of $8.06 \%$ at locations, and on the other hand, a location has, on average, a recurrence ratio of $13.53 \%$. For a category at the top level (10 totally), the average recurrence ratio is $10.7 \%$. On average, people's visits have a highest recurrence ratio on location of $37.4 \%$ (by $90 \%$ people). Normally, people have a highest recurrence ratio of $6.9 \%$ to $71.9 \%$ on location.

For different US cities, the average recurrence ratio at location are distinctive. For example, taking New York as an example of eastern cities in US, people have an average recurrence ratio at location of $6.96 \%$ which is far below the average ratio of US, while taking Los Angeles as an example western city in US, people have an average recurrence ratio of $8.71 \%$ which is obviously more than it in US.

Table 1. The average recurrence ratio at location.

\begin{tabular}{|l|l|l|l|}
\hline $\begin{array}{l}\text { Average recurrence } \\
\text { ratio }\end{array}$ & US (\%) & NY (\%) & LA (\%) \\
\hline individuals & 8.06 & 6.96 & 8.71 \\
\hline locations & 13.53 & 7.27 & 8.67 \\
\hline categories & 10.70 & 6.88 & 9.05 \\
\hline
\end{tabular}

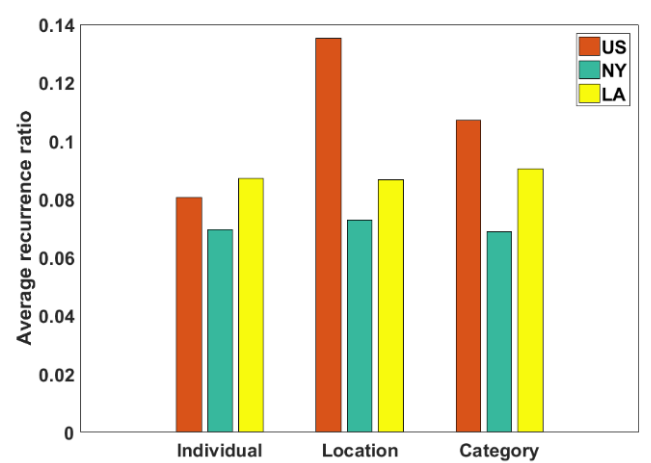

Figure 3. The average recurrence ratio at location.

The average recurrence ratios of different categories are summarized in Table 2 and Figure 4. For example, for the category Food, the average recurrence ratio at location is $22.65 \%$ which suggests that people go to a same restaurant with an average recurrence ratio between $1 / 4$ and $1 / 5$ of their daily travel. The highest ratio is Residence with an average recurrence ratio of $26.10 \%$ while the lowest ratio is Event with $3.37 \%$. For different cities, average recurrence ratio at location are different from each other. For example, people have a higher average 

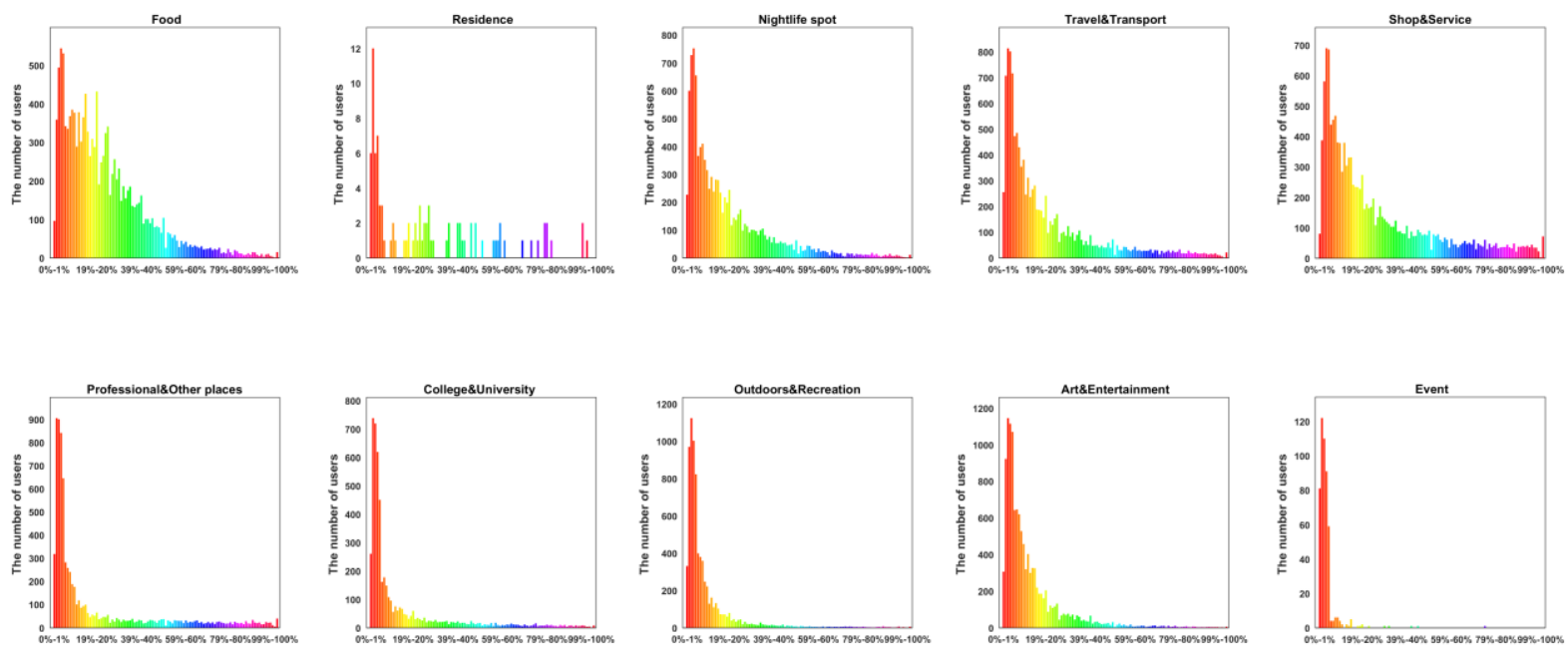

Figure 5. Distributions of recurrence ratios on different categories of locations.

recurrence ratio of $32.00 \%$ in Los Angeles rather than in US, for locations on Food.

Table 2. The average recurrence ratio at locations on different categories.

\begin{tabular}{|l|l|l|l|}
\hline $\begin{array}{l}\text { Average recurrence } \\
\text { ratio on categories }\end{array}$ & US (\%) & NY (\%) & LA (\%) \\
\hline Food & 22.65 & 29.33 & 31.99 \\
\hline Residence & 26.10 & 13.82 & ----- \\
\hline Nightlife Spot & 18.27 & 17.30 & 16.70 \\
\hline Travel\&Transport & 19.39 & 18.18 & 16.99 \\
\hline Shop\&Service & 25.88 & 20.24 & 25.77 \\
\hline $\begin{array}{l}\text { Professional } \\
\text { \& Other Places }\end{array}$ & 18.94 & 12.68 & 15.65 \\
\hline $\begin{array}{l}\text { College } \\
\text { \& University }\end{array}$ & 13.84 & 9.50 & 12.08 \\
\hline $\begin{array}{l}\text { Outdoors } \\
\text { \& Recreation }\end{array}$ & 8.96 & 11.46 & 9.64 \\
\hline $\begin{array}{l}\text { Arts } \\
\text { \& Entertainment }\end{array}$ & 11.99 & 13.22 & 14.14 \\
\hline Event & 3.37 & 4.77 & ----- \\
\hline
\end{tabular}

We also investigate whether there exists difference between the recurrence ratios on different categories of locations by illustrating the distributions of the ratios with respect to percentages of people in US (Figure 5). We observe that for different categories, the distribution curves of average recurrence ratio at location are also distinctive. For example, the recurrence ratio on category Food follows a Gauss distribution with a gentle slope, while it on category Outdoors \& Recreation presents a sharp distribution. From the results, we conclude that the recurrence ratio on location reveals that people's behavior preference on different categories is diverse, i.e. some categories tend to be visited very frequently by people with relatively stable preference, while some categories are visited very occasionally.

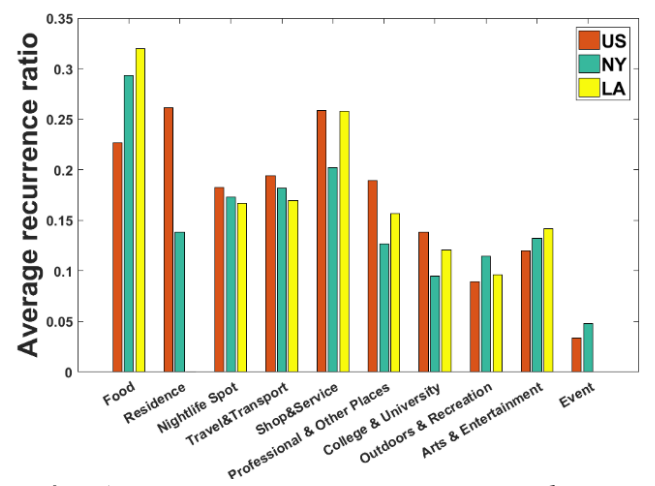

Figure 4. The average recurrence ratio at locations on different categories.

\subsection{The activity of people's daily travel}

Then we give a novel measure activity to justify the frequency degree of people's visiting on locations. For people in a city, different locations signify distinctive meanings in their daily life, some locations are visited frequently and routinely, some are visited occasionally. Therefore, for an individual in a city, we define another measure called the number of certain locations by counting the number of locations visited by him/her with recurrence ratios beyond the average recurrence ratio at location. Similarly, we define an opposite measure called the number of certain locations by counting the number of locations visited by him/her with recurrence ratios below or equal to the average recurrence ratio at location. 

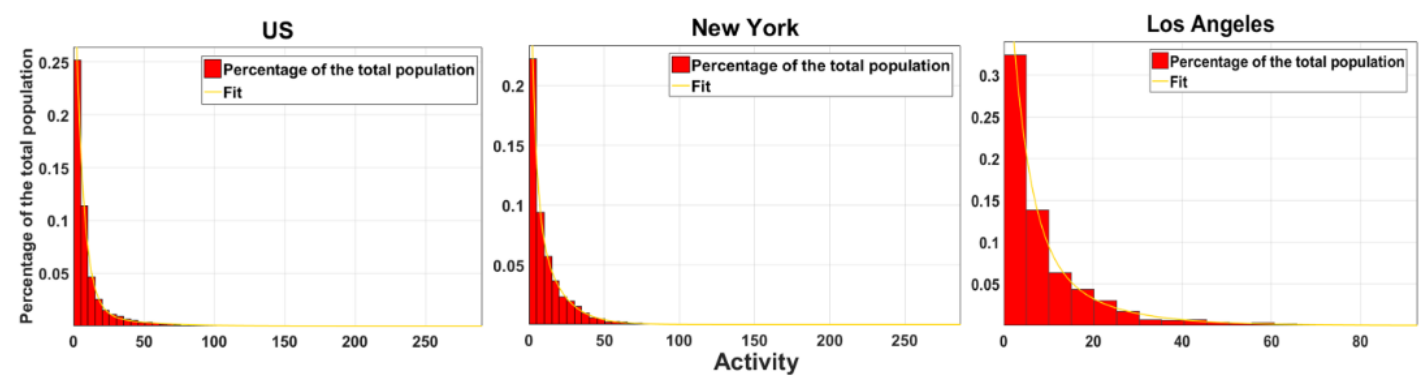

Figure 6. Distribution of Activity of people's daily travel in US.

Certain_loc $i j=$ \# locations visited by individual $j$ in city $i$ with recurrence ratios beyond Ave_Recur ${ }_{i}$

Uncertain_loc $c_{i j}=$ \# locations visited by individual $j$ with recurrence ratios below or equal to Ave_Recur ${ }_{i}$

Further, we use the calculated average Ave_Recur ${ }_{i}$ as a measure, to further count number of locations visited by him/her with a frequency higher than Ave_Recur $i$, denoted as Certain_loc $i j$, and that with a frequency equal or lower than $\overline{A v e}$ Recur $i$, denoted as Uncertain_loc $i j$. Next, we define the measure activity for each individual as Uncertain_loc $i j$ versus Certain_loc $c_{i j}$, denoted by Activity ${ }_{i j}$.

$$
\text { Activity }_{i j}=\text { Uncertain_loc }_{i j} \text { versus Certain_loc } i j
$$

Activity $_{i j}$ reflects the degree of activity of an individual $j$ 's out-door behavior in terms of locations in city $i$. A high Activity $_{i j}$ suggests that an individual tends to visit more locations with relative lower frequencies rather than locations with relative high frequencies. So we may think the out-door behavior of the individual is somewhat uncertain, for instance, the daily life of a popular movie star. A low Activity suggests that an individual tends to visit more locations with relative high frequencies rather than locations with relative low frequencies. So we may think the out-door behavior of the individual is somewhat stable, for instance, the daily life of a busy middle school teacher. Thus Activity reflects activity degree of people's out-door behavior. We illustrate the distributions of Activity of people's daily travel patterns in US and some typical US cities (Figure 6).

We also investigate average activity of people's daily travel in 10 typical US cities and summarized the results in Figure 7 and Table 3. On average, Activity of people's daily life in US is around 5.00. Difference average Activity are observed in terms of different US cities.

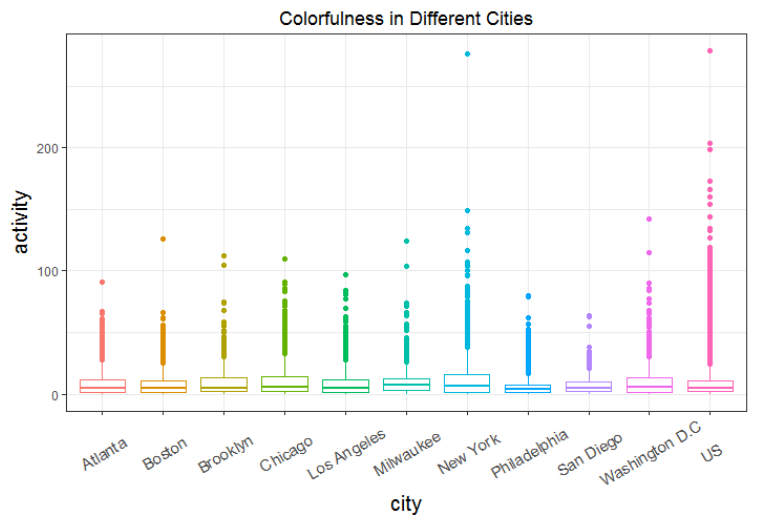

Figure 7. Activity of people's daily travel in US cities.

Table 3. Average Activity of people's daily travel in US cities.

\begin{tabular}{|l|r|r|r|}
\hline Area & Median & Mean & Std. \\
\hline Atlanta & 5.16 & 9.06 & 11.53 \\
\hline Boston & 5.00 & 9.13 & 12.22 \\
\hline Brooklyn & 5.00 & 8.76 & 10.58 \\
\hline Chicago & 6.00 & 11.07 & 14.64 \\
\hline Los Angeles & 5.00 & 8.64 & 11.18 \\
\hline Milwaukee & 7.00 & 10.24 & 12.39 \\
\hline New York & 6.33 & 11.39 & 15.16 \\
\hline Philadelphia & 3.66 & 6.70 & 9.71 \\
\hline San Diego & 4.50 & 7.41 & 8.79 \\
\hline Washington D.C & 5.33 & 10.01 & 13.75 \\
\hline US & 5.00 & 9.97 & 14.09 \\
\hline
\end{tabular}

\section{Study 2: Is people's daily lifestyle colorful?}

This question is addressed by evaluating people's daily lifestyles, from their observed daily travel pattern. Therefore, we develop another basic measure named the recurrence ratio on categories to find people's repetitiveness of their daily travel in term of location categories. Then we give an advanced novel measure colorfulness to investigate if people's daily lifestyle is colorful. 

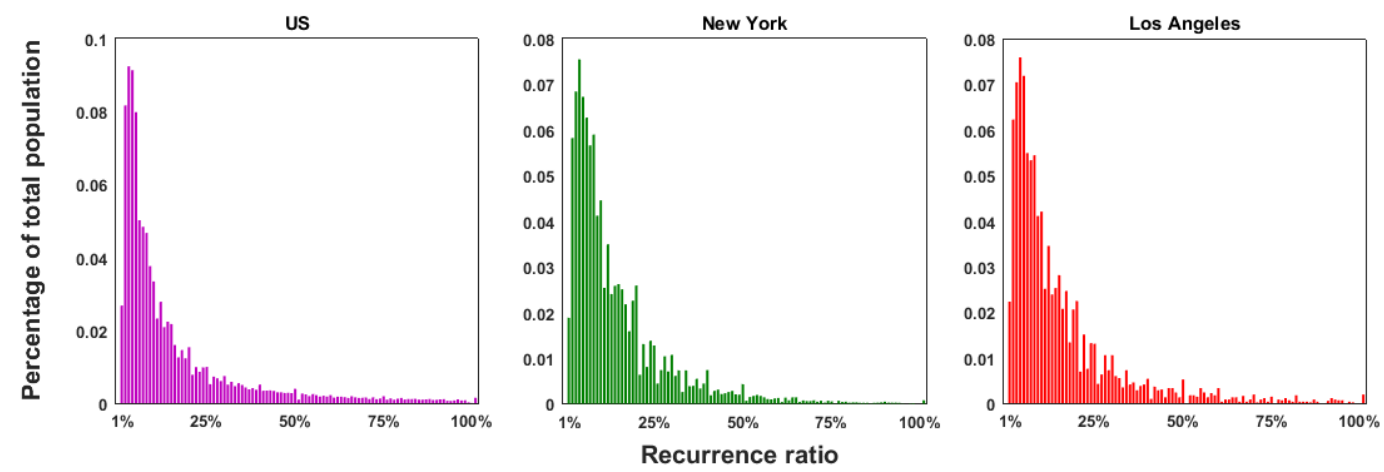

Figure 8. Distribution of the average recurrence ratio on category in US.

\subsection{The recurrence ratio on category}

We define the recurrence ratio on category as the percentage of an individual's visits on a certain location category among all his/her visiting records. By calculating numbers of categories an individual's visited in a certain interval of the recurrence ratio (e.g. $5-10 \%$ ), and to average the numbers of categories in each interval for all people, we get the average recurrence ratio by category. As for all people in US cities, we illustrate the distribution of average recurrence ratio on category in Figure 8 .

First, we calculate numbers of categories where an individual visit at a frequency within a certain repetition internal. Then, people who visited at least one category within the recurrence ratio interval are added. Finally, we obtain the complete distribution at each interval of the recurrence ratio by category. On average, people visit about 4 location categories within the interval of recurrence ratio $5-10 \%, 2$ categories within the internal of $10-15 \%$, and 1 category within the internals of $4-5 \%$ and $15-20 \%$, respectively, in US. We find the recurrence ratio by category follow a Gauss distribution in terms of the number of location categories in each US city. Results demonstrate that there exist common patterns over all cities in US. On average, people's visits have a highest recurrence ratio on category of $56.0 \%$ (by $90 \%$ people). Normally, people have a highest recurrence ratio of $31.1 \%$ to $85.7 \%$ on categories (10 categories at the top level) and a highest recurrence ratio of $21.2 \%$ to $78.6 \%$ on categories (281 categories at the second level). On average, people's visits have a highest recurrence ratio on category of $56.0 \%$ (by $90 \%$ people).

\subsection{The colorfulness of people's daily lifestyles}

We propose a novel measure colorfulness to justify the diversity degree of people's daily travel. Similar to the measure activity, first, according to the distribution of recurrence ratio by category for people in a certain area, we define an exact percentage Ave_Recur_cate, called average recurrence ratio by category, under which the summarized number of categories of an individual's daily travel with the recurrence ratios equal or below Ave_Recur_cate , is as the same as the number of categories with the recurrence ratios beyond Ave_Recur_cate. We then call Ave_Recur_cate ${ }_{i}$ as the average recurrence ratio by category for an individual in the area. By averaging Ave_Recur_cate $i$ for all people in the area, we get the average recurrence ratio by category for the area.

Certain_cate ${ }_{i j}=$ \# categories visited by individual $j$ in city $i$ with recurrence ratios beyond Ave_Recur_cate

$\bar{U}_{\text {ncertain_cate }} i j=\#$ categories visited by individual $j$ with recurrence ratios below or equal to Ave_Recur_cate $i$

Then, for an individual $j$, we use Ave_Recur_cate as a measure, to further count the number of categories visited by him/her with a visiting frequency higher than Ave_Recur_cate $e_{i}$, denoted as Certain_cate $e_{i j}$, and that with a frequency equal or lower than

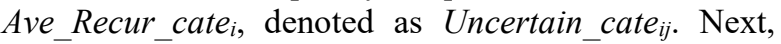
we define the measure colorfulness for each individual in city $i$ as Uncertain_cate $e_{i j}$ versus Certain_cate $i j$, and denoted by Colorfulness $i j$.

$\underset{\text { Certain_cate }_{i j}}{\text { Colorfulness }_{i j}}=\quad$ Uncertain_cate $_{i j} \quad$ versus

Colorfulness reflects the degree of diversity of an individual's daily lifestyle in terms of categories. We investigate the distribution of Colorfulness in terms of the percentage of total population in US (Figure 9). A high colorfulness suggests that an individual tends to visit relatively more location categories with low frequencies, rather than categories with high frequencies. So we may think the daily lifestyle of the 

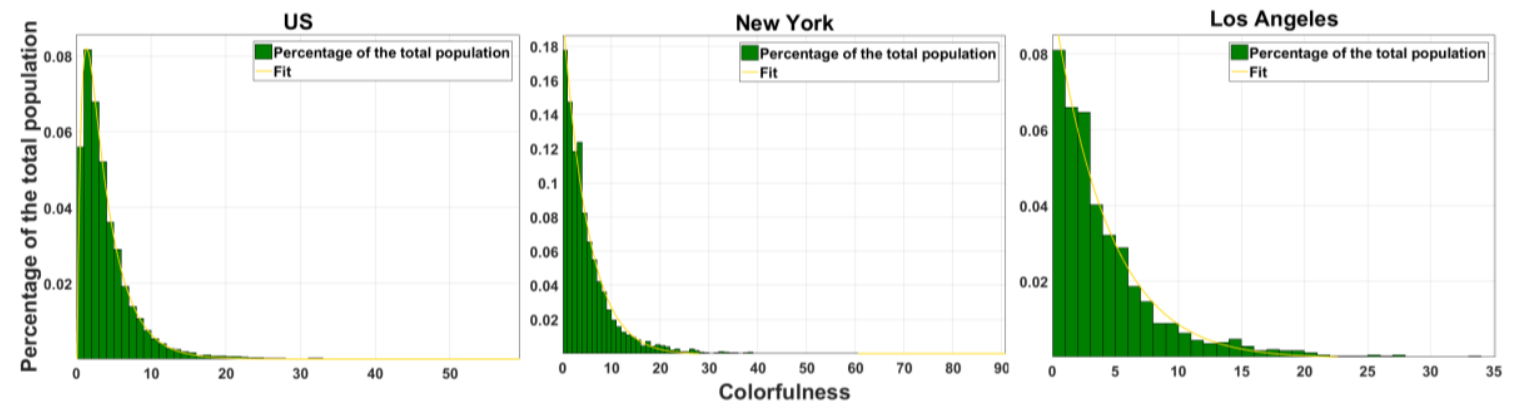

Figure 9. Distribution of Colorfulness of people's daily life in US cities.

individual is somewhat diverse, for instance, a traveler. A low colorfulness suggests that an individual tends to visit relatively more categories of locations with high frequencies rather than those with low frequencies. So we may think the daily lifestyle of the individual is somewhat monotonous, for instance, a busy postman. Thus colorfulness exactly reflects diversity degree of people's daily lifestyle.

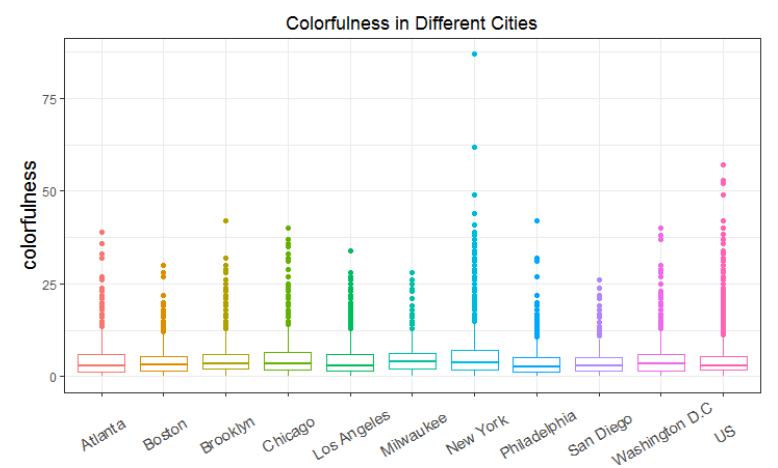

Figure 10. Colorfulness of people's daily lifestyle in US cities.

Table 4. Average Colorfulness of people's daily lifestyle in US cities.

\begin{tabular}{|l|r|r|r|}
\hline Area & Median & Mean & Std. \\
\hline Atlanta & 3.00 & 4.33 & 4.68 \\
\hline Boston & 3.25 & 4.23 & 4.13 \\
\hline Brooklyn & 3.33 & 4.74 & 4.45 \\
\hline Chicago & 3.50 & 4.85 & 4.89 \\
\hline Los Angeles & 3.00 & 4.34 & 4.44 \\
\hline Milwaukee & 4.00 & 4.83 & 4.16 \\
\hline New York & 3.66 & 5.24 & 5.81 \\
\hline Philadelphia & 2.66 & 4.00 & 4.33 \\
\hline San Diego & 3.00 & 3.99 & 3.95 \\
\hline Washington D.C. & 3.33 & 4.64 & 4.94 \\
\hline US & 3.00 & 4.23 & 4.05 \\
\hline
\end{tabular}

We also illustrate the average colorfulness in different US cities and summarize the results in Table 4 and Figure 10. Most people have the colorfulness within 20. On average, Colorfulness of people's daily lifestyle in US is around 4.35. Besides, we observe distinctive colorfulness in different US cities. For example, taking New York as a typical eastern city in US, people have an average colorfulness of 5.6 which is much higher than it of US population, while taking Los Angeles as a typical western city, people have an average colorfulness of 4.7 which is obviously lower than it of US population. There is significant difference on colorfulness between people.

\section{Study 3: Does activity or colorfulness disclose people's preference?}

We further investigate whether different groups of people in terms of activity in daily travel or colorfulness of lifestyle also have significantly distinctive preference. For the purpose, we illustrate people's profiles of different groups of people on travel patterns evaluated by activity and on lifestyles in terms of colorfulness, respectively. By comparing and illustrating profiles using their total historical daily travel records tagged with location categories, we find that there exists distinctive location preference among people with different values of activity and colorfulness.

\subsection{Does activity disclose people's preference?}

We first investigate whether people's preferences are significantly distinctive. For people with high activity and those with low activity, we illustrate their preference profiles via tags on location categories, respectively, to investigate whether the preference on categories significantly distinctive and illustrate the results in Figure $11(a, b)$.

By comparing and illustrating profiles of people with high and low activity in US cities, we find that there exists distinctive preference among people with different values of activity. People with high activity tend to have diverse and obvious preference, while people with low activity have unobvious preference. As for female whose activity are relatively high, typical location tags are Bar, Coffee Shop, Gym/Fitness center, Park and Stadium, while male with high activity, typical tags are Bar, Airport, 


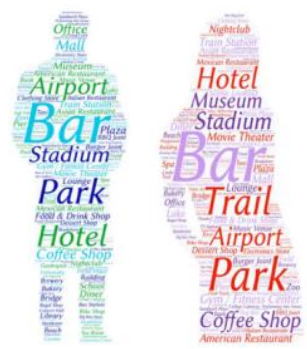

(a) Active group (US).

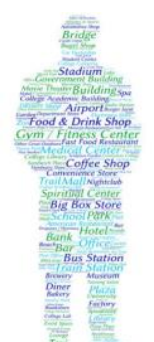

(b) Inactive group (US)

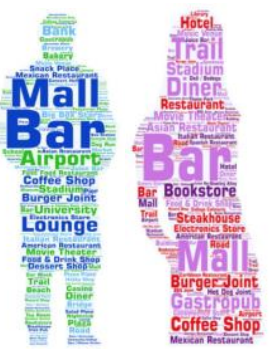

(c) Colorful life style (LA)

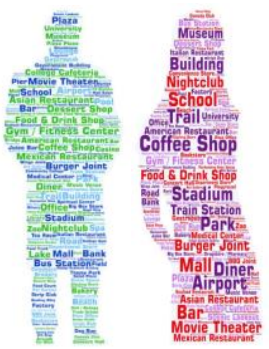

(d) Monotonous life style (LA)

Figure 11. Profiles of people with respect to their daily travel's activity and colorfulness.

Stadium, Gym/Fitness center, American Restaurant and Coffee Shop. This finding indicates that if a female is active in her daily travel, she is probably with many friends, has frequent parties and likes sports as well, while if a male has an active daily travel pattern, he probably a busy working man who has frequent travels, likes sports and fast food.

Female and male cannot be distinguished obviously if their activity are lower than average. However, by performing a statistical analysis on the top 10 tags of locations, we found that female who are inactive tend to go to Government Building, Bar, Food \& Drink Shop, while male who are inactive like to go to Gym/Fitness center, Food \& Drink Shop, Medical Center, Airport. Food \& Drink Shop are the common tags of locations for inactive male and female, which disclose inactive people are interested at food. Government Building is ranked at the top 1 tag for inactive female, which suggests that if the daily travel pattern of a female is inactive, she is probably busy working. Medical Center is ranked top 2 for male, which reveals that if a male is lazy in his daily travel, he is probably ill or has a high probability to get ill. People with high activity are also tagged with diverse tags in different cities, which suggests that city characteristics also influence people's daily travel.

\subsection{Does colorfulness disclose people's preference?}

For people with high colorfulness and those with low colorfulness, we illustrate their preference profiles via tags on location categories, respectively, to investigate whether the preference on categories significantly distinct and illustrate the results in Figure $11(\mathrm{c}, \mathrm{d})$.

We observe that there exists distinctive preference on location category. For instance, people whose daily life are colorful with an activity value more than 20 in US tend to go to Bar, Park, Hotel, Stadium and such entertainment places as Mall, Plaza and Museum, while people who are inactive with an Activity value less than 1 in US have no obvious preference on locations.

We then investigate whether people's preference on different types of locations are significantly distinctive. By comparing and illustrating profiles of people with high and low colorfulness, we find that there exists distinctive preference among people with different colorfulness. People with high colorfulness tend to have diverse and obvious preference, which suggests that city characteristics also influence people's daily lifestyle.

As for female whose colorfulness are relatively high, typical location tags are Bar, Stadium, American Restaurant, Airport, while male with high colorfulness, typical tags are Bar, Train Station, Gym/Fitness Center, Stadium and Airport. This finding indicates that if a female has a colorful lifestyle, she is probably rich in heart, while if a male's lifestyle is colorful, he is probably a tourist or sports enthusiast.

People with low colorfulness have relatively unobvious preference. Female and male cannot be distinguished obviously if their colorfulness are lower than average. For both female and male who have monotonous life styles, top 5 location categories are exactly the same which are Bar, Gym/Fitness Center, Airport, Coffee Shop and Food \& Drink Shop.

We also observe that there exists distinctive preference on location category. For instance, people whose daily life are colorful with a Colorfulness value more than 50 in US tend to go to Bar, Park, Hotel, Stadium and such entertainment places as Mall, Plaza and Museum, while people whose daily life are very monotonous with a Colorfulness value less than 1 in US have no obvious preference on categories.

\section{Conclusions}

We propose two basic measures and two advanced metrics evaluating people's daily travel patterns and lifestyles, respectively, on the basis of extracted historical records of people's observable daily travel and trajectories from a location-based social network, 
Foursquare. We conduct statistical analysis and comparison and illustrate distributions of the proposed measures for populations in US cities. By illustrating profiles of groups of people on the basis of the developed measures, we find citizens' preference can be distinguished well. Results also show both consistent and opposite findings to common sense, in terms of people's travel pattern and lifestyle.

Specifically, Study 1 defines a novel metric to measure people's daily travel pattern, namely recurrence ratio at location which indicates basic repetitiveness of daily visits. Results show that in a certain area, people's recurrence ratio on locations follows an exponential distribution. A novel metric Activity is then developed on the basis of recurrence ratio on locations, which reveals the tendency of people's behavior patterns in terms of determinacy or non-determinacy. Results show that people's activity follows a power-law distribution in a certain area. Findings also indicate that people have distinctive activity of daily travel patterns in different cities.

Study 2 begins by defining another novel metric to measure people's daily travel pattern, namely recurrence ratio on category which indicates basic repetitiveness of different locations. Results show that in a certain area, people's recurrence ratio on category follows a Gauss distribution, which is different from the distribution of the recurrence ratio at locations. A novel metric colorfulness is then developed on the basis of recurrence ratio on category, which reveals the characteristics of people's daily lifestyle related with diversity or monotony. Results show that colorfulness follows a strict power-law distribution in a certain area. Distinctive colorfulness of daily lifestyle is found among different US cities.

Study 3 investigates whether different groups of people in terms of activity in their daily travel or colorfulness of their lifestyle also have significantly distinctive preference. We give profiles of different groups of people on travel patterns evaluated by activity and on lifestyles in terms of colorfulness, respectively. By comparing and illustrating profiles using their total historical daily travel records tagged with location categories, we find that there exists distinctive location preference among people with different values of activity and colorfulness. People with high activity and colorfulness tend to have diverse and obvious preference, while people with low activity and colorfulness have unobvious preference. Preference are obviously distinctive between people who have stable daily travel pattern and those who have uncertain and occasionally travel pattern, as well as people whose lifestyle are colorful and those whose are monotonous.
Gender is found as a determinate factor which helps to illustrate people's profiles on location preference, on the basis of their activity and colorfulness.

Findings demonstrate that the proposed measures are supposed to be effectively adopted for researchers on trajectory prediction, preference analysis, location recommendation, and further give suggestions to city managers and business fields for decision making.

This study gives directions and suggestions not only for developed countries but also for developing countries to enhance their online personalization service and promote related business through information technology on the basis of location-based social media. Future research may pay more attention on the relativeness between people's daily travel patterns and their financial behaviors to explore and explain more interesting social phenomena.

\section{Acknowledgement}

This work is partly supported by the National Natural Science Foundation of PRC (No. 71471016, 71531013, 71729001).

\section{References}

[1] Al-Shamri, M. Y. H. 2016. "User Profiling Approaches for Demographic Recommender Systems," KnowledgeBased Systems (100), pp. 175-187.

[2] Guo, G., Zhang, J., Zhu, F., and Wang, X. 2017. "Factored Similarity Models with Social Trust for Top$n$ Item Recommendation," Knowledge-Based Systems (122), pp. 17-25.

[3] Jia, D. W., Zeng, C., Peng, Z. Y., Cheng, P., Yang, Z. M., and Lu, Z. 2012. "A User Preference Based Automatic Potential Group Generation Method for Social Media Sharing and Recommendation," Chinese Journal of Computers (35:11), pp. 2381-2391.

[4] Lee, W. P., and Ma, C. Y. 2016. "Enhancing Collaborative Recommendation Performance by Combining User Preference and Trust-Distrust Propagation in Social Networks," Knowledge-Based Systems (106), pp. 125-134.

[5] Liu, Q., Wu, S., Wang, L., and Tan, T. 2016. "Predicting The Next Location: A Recurrent Model with Spatial and Temporal Contexts," Thirtieth AAAI Conference on Artificial Intelligence, pp. 194-200, AAAI Press.

[6] Zhang, J., Zheng, Y., Qi, D., Li, R., and Yi, X. 2016. "DNN-Based Prediction Model for Spatio-Temporal Data," Proceedings of The 24th ACM SIGSPATIAL International Conference on Advances in Geographic Information Systems (2016:92), ACM. 
[7] Cheng, C., Yang, H., Lyu, M. R., and King, I. 2013, August. "Where You Like to Go Next: Successive Point-of-Interest Recommendation," In Proceedings of the Twenty-Third International Joint Conference on Artificial Intelligence, pp. 2605-2611, AAAI Press.

[8] Huang, J. B., Zhang, P. P., Huang, F., Xue, J., and Sun H. 2014. "A Trajectory Prediction Approach for Mobile Objects by Combining Semantic Features," Journal of Computer Research and Development (51:1), pp. 76-87.

[9] Liu, S., Li, G., Tran, T., and Jiang, Y. 2016. "Preference Relation-Based Markov Random Fields for Recommender Systems," Machine Learning, pp. 1-24.

[10] Ye, J., Zhu, Z., and Cheng, H. 2013. "What's Your Next Move: User Activity Prediction in LocationBased Social Networks," In Proceedings of the SIAM International Conference on Data Mining, pp. 171-179, SIAM.

[11] Yu, F., Liu, Q., Wu, S., Wang, L., and Tan, T. 2016. "A Dynamic Recurrent Model for Next Basket Recommendation," The, International ACM SIGIR Conference, pp. 729-732, ACM.
[12] Yu, Y., and Chen, X. 2015. "A Survey of Point-ofInterest Recommendation in Location-Based Social Networks," AAAI Workshops-Trajectory-Based Behavior Analytics, pp. 53-60.

[13] Cho, E., Myers, S. A., and Leskovec, J. 2011. "Friendship and Mobility: User Movement in LocationBased Social Networks," ACM SIGKDD International Conference on Knowledge Discovery and Data Mining, San Diego, CA, USA, August, pp. 1082-1090.

[14] Hariri, N., Mobasher, B., and Burke, R. 2013. "QueryDriven Context Aware Recommendation," In Proceedings of the 7th ACM Conference on Recommender Systems, pp. 9-16, ACM.

[15] Jamali, M., and Ester, M. 2010. "A Matrix FactorizationTechnique with Trust Propagation for Recommendation in Social Networks," ACM Conference on Recommender Systems, Recsys 2010, Barcelona, Spain, September (Vol.45), pp. 135-142.

[16] Ma, H., Zhou, D., Liu, C., Lyu, M. R., and King, I. 2011. "Recommender Systems with Social Regularization," Forth International Conference on Web Search and Web Data Mining, WSDM 2011, Hong Kong, China, February, pp. 287-296. 